\title{
Advanced Peak Shaving Control Strategies for Battery Storage Operation in Low Voltage Distribution Network
}

\author{
Jernej Zupančič, Edin Lakić, Tomi Medved, and Andrej F. Gubina \\ Laboratory of Energy Policy \\ University of Ljubljana, Faculty of Electrical Engineering \\ Ljubljana, Slovenia \\ Jernej.Zupancic@fe.uni-lj.si
}

\begin{abstract}
The increased integration of PV systems in the low voltage network causes many problems in network operations. The EU project STORY is tackling these problems with adding energy storage to the grid. In this paper we present new control concept with peak demand control algorithm which is used on storage unit in $\mathrm{LV}$ distribution network. The algorithm development is based on real measurements from transformer station and inclusion of solar irradiation forecast. On the basis of forecasts algorithm defines new threshold levels of power flows through transformer which is responsible for decision of battery charge/discharge related to power surplus in real-time operation. Results of the simulations show an improvement in the operation of peak shaving control.
\end{abstract}

Index Terms - electricity storage, battery, control strategies, peak shaving, low voltage network.

\section{INTRODUCTION}

The deployment of renewable energies (RES) in the power grid has seen a strong interest recently due to beneficial support policies applied around the world [1]. In distribution network, grid-connected photovoltaic (PV) installations have grown radically. Under high PV penetration levels, large reverse power flow may lead to voltage rise, which causes potential issues to the distribution network [2]. The integration of increasing shares of RES into the grid creates challenges to the stability and reliability of the power system. Production and consumption of electricity need to be in balance at all times, and the fluctuating nature of the energy produced by RES units makes this task difficult, creating stability issues to the power system. Stationary energy storage systems (ESSs) can advantageously be used in the modern electricity networks, providing the necessary flexibility to the power system.

One of the solutions to tackle problems of high peak demand and grid instability due to high PV power penetration is to deploy battery energy storage systems (BESSs) to support the operation of public distribution grids [3]-[9]. BESSs technologies are expected to help overcoming such issues in electricity grids since they are able to support the efficient integration of Distributed Energy Resources (DERs), provide ancillary services, emulate grid inertia, allow off-grid operation, electrify remote areas, enhance power quality and allow for postponing of grid investment [10]-[12]. BESS installed in low voltage (LV) distribution network can provide functions such as peak shaving and valley filling (i.e. charge when demand is low and discharge when demand is high).

Solar PV generation is dependent on the inclination of incoming solar radiation; hence, peak generation occurs during the middle of the day, typically when demand in the residential distribution network is low. Due to the behaviour of consumers (i.e. energy demand activities in the evening) and the nature of solar PV generation, there is an incongruity between energy generation and its consumption [13]. To be effective, BESSs scheduling systems must ideally charge during periods of high PV generation or low demand and discharge during periods of peak demand. Many methods tackle these problems, e.g. using the optimisation algorithms to minimise or maximise objective functions or finding optimal solution through dynamic programming, [14]-[16].

Even though the BESSs can buffer the gap between the volatile energy production from $\mathrm{PV}$ and the consumption of energy there are still needs for infrastructure in electricity grid and for reliable demand and generation profiles' forecast. For example, on a sunny day the battery is often fully charged before the peak of the PV production is reached. Increasing the size of the battery does not necessarily help to solve this problem as beyond a certain capacity, it will not be discharged sufficiently by the local demand over-night. Therefore, many different solutions have been proposed using voltage control strategies, and management algorithms for peak shaving with BESSs [17]-[21].

This paper describes the endeavours of the European H2020 project STORY which focusses on the added value that thermal and electrical storage can bring, showing the inter-relations between technologies and stakeholders as well as the potential and impact of policy and regulation. In our paper we investigate 
the peak shaving and optimisation of the medium-scale battery storage integrated into the distribution grid. The simulations cover the LV network of the STORY residential demo site Suha in Slovenia. Later in the project the results will be verified in real-life demonstration.

The paper is organised as follows. It starts with a description of chosen key grid challenges tackled within the STORY project. In Section III a test network is described together with a short description of the proposed hierarchical control concept. Section IV describes the peak shaving algorithm. The simulation test cases are given in Section V. Simulation study results together with discussion and recommendations for future work are provided in Section VI. The paper concludes with the final findings in Section VII.

\section{KEY GRID CHALLENGES}

In the STORY project, the following key grid challenges pertaining to the distribution network have been selected:

- $\quad$ Security of electricity supply (SoES),

- Active power reserve provision;

- Voltage control; and

- Current congestion mitigation.

Since several aspects of the SoES are provided using the operating reserve in the power system, we have therefore included active power reserve provision among the aspects of the SoES key grid challenge.

Security of supply broadly covers several aspects. In the long-term of operation, SoES consists of access to primary energy sources for electricity production, system adequacy or ability of the system to convert primary fuels into electricity and transmit that electricity to end users in a sustainable manner. On one hand, it implies generation adequacy, defined as the presence of sufficient generating capacity to meet demand (both in base load and in peak periods) taken together with imports of electricity. On the other hand, system adequacy includes network adequacy which is the ability of sufficient network infrastructure to meet demand. The last point in long-term security of supply is market adequacy, which is the ability of the market to facilitate the link between the producers and consumers of electricity.

In short-term security of supply, operational reliability and security of the system as a whole and its assets, including the ability to overcome short-term failures of individual components of the system is investigated.

Voltage control in Distribution System operation is keeping steady state voltage levels within acceptable ranges, defined by regulatory framework and operation standards. Voltage levels are maintained with direct control of voltage or through control of reactive power flow, which affects the voltage drop. The equipment normally used for the voltage and reactive power control are installed on load tap charger transformers, switched shunt capacitors and step voltage regulators. Conventional distribution networks are designed based on the assumption of unidirectional power flow, where active power flows from the higher voltage level towards the lower voltage level networks, and the voltage decreases along the feeder from the substation to the remote end. With the increasing share of DRES in the distribution network, voltage control faces new challenges of bi-directional power flows in the network and rising local voltage magnitudes in proximity of DRES location, both caused by excessive local generation.

Another issue regarding voltage control is overvoltage, which is defined as the peak value of voltage exceeding the corresponding peak of the highest voltage for equipment. They can be caused by switching manoeuvres, electrostatic discharge, atmospheric origins and power frequency overvoltage, associated with permanent state in the network or injection of high amount of power.

Current congestion mitigation prevents overloading of the network elements, which could result in increased loss rate and damage of the element due to unexpected high current levels. Congestion in distribution networks can occur as a result of the high penetration of distributed energy resources and low load conditions. Current congestion problems in distribution networks are envisaged as voltage problem (which means that bus voltage is close to or exceeding the limit) and overloading problem (loading is close to or exceeding the thermal limit of the power components).

\section{NETWORK ANALYSIS AND HIERARCHICAL CONTROL CONCEPT DESCRIPTION}

In order to investigate three key grid challenges identified in the previous section, a medium scale storage unit is to be installed in the transformer substation of residential low voltage network of EG within the STORY project. To be able to maximize the utilization of the BESS, a hierarchical control is proposed which would enable an operation of different services and control algorithms.

\section{A. Network description}

The simulations were done for low voltage network residential demo site Suha village which is located in northwest Slovenia. Medium scale storage unit for our demo case is connected to $20 / 0.4 \mathrm{kV} \mathrm{MV} / \mathrm{LV}$ transformer station supplying Suha village residential grid in the vicinity of Elektro Gorenjska (EG), Slovenian distribution company which is located in Kranj. The SUHA village LV network is an example of a 0.4 $\mathrm{kV}$ rural cable network (no overhead lines) with high penetration of PV generation. There are 7 dispersed PV plants installed in Suha LV network and 78 household metering points. The demo site is already sufficiently equipped with meters, controllers and communications. The medium scale BESS will be integrated into the grid for peak shaving and optimization. The application requires measurement of many electrical parameters including power quality at houses (consumers and PV producers) used for control algorithm for optimization of battery storage. Therefore, new meters and controllers for storage are required and will be interconnected locally and to control centre.

\section{B. Hierarchical control concept description}

To tackle the key grid challenges in the network, a threelayered control was designed to control the storage system, as seen in Figure 1. Primary or Device control layer is located directly in device, Secondary or Site control layer supervises multiple devices, located at one location, and Tertiary, Remote control layer manages multiple sites, spread across network. 


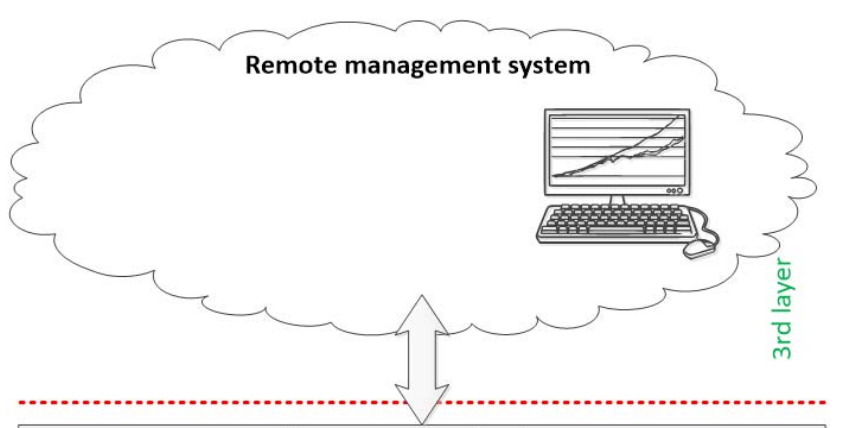

Site management system

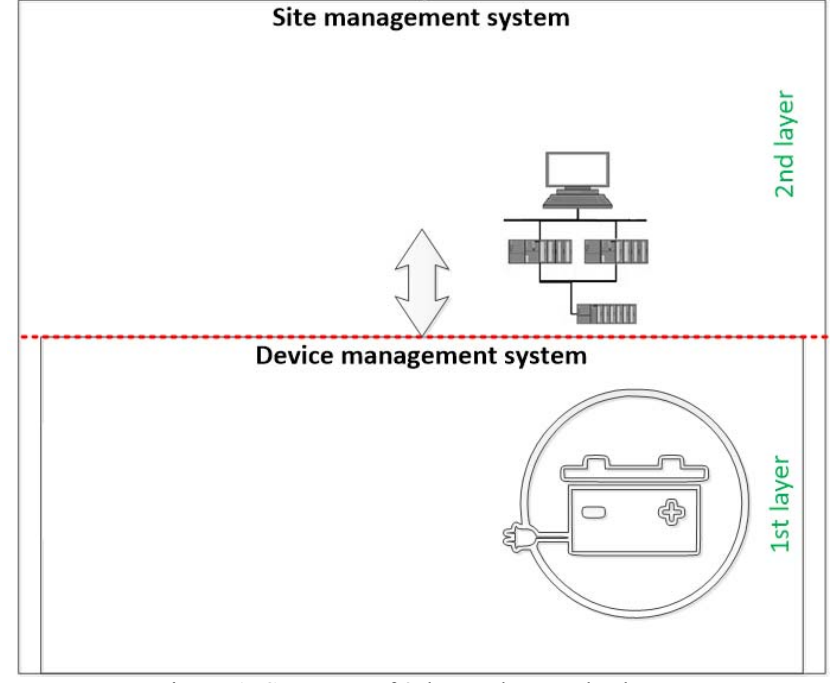

Figure 1: Structure of 3-layered control scheme

The Primary control layer, device control, is built-in and enables normal operation of the storage system via system activators and sensors. Besides executing the commands received from higher layers, it also provides information about system status, levels of charge and thermal information, which all influence and determine the operating modes of the system.

Site management system, which represents the Secondary control layer, is responsible for control mechanisms, which determine the operating mode of the system and sending information to primary layer. Based on the available data, the control algorithm decides how storage system is utilised. This layer is designed to be self-sufficient in case of the tertiary control layer outage or inaccessibility.

The Tertiary control layer is the remote control system, which is situated at central control site of the operator. Control strategies and optimisation methods of the system operation are performed here. Additional measurements, which are performed system-wide are collected here and influence the selection of the control strategy along with information about weather situation, demand and production forecasts in the system, electricity prices, contracts and other defined schedules.

\section{PEAK DEMAND CONTROL AlgORITHM}

For the demonstration site in residential LV grid, multiple Use Cases (UC) were proposed. Controls, located in the first layer of control, are responsible for safety and device control and can provide Voltage Control (VC) and Fault ride through (FRT) capability. In the second layer, where we have Site management controls, a programmable Remote Terminal Unit (RTU) is communicating with $1^{\text {st }}$ and $3^{\text {rd }}$ layer of control. On this unit we have several control algorithms, which enable additional UC.

Peak Demand Control (PDC) is a combination of peak shaving and peak shifting functionality, where storage activation compensates Transformer station peak loads and peak production of renewables in the network.

Reserve Provision is an ancillary service offered to transmission and distribution system operators (TSO, DSO), where storage, which is predefined in amount and duration of provision in the contract, is utilized when the customer needs or wishes to use the available energy.

Zero Load Operation is activated in order to minimize the power flows through the transformer station, and achieve island-mode like situation of the LV network. With Reactive Power Compensation storage is activated to minimize the reactive power flows from the MV grid in order to reduce the losses in transformer substation.

In this particular LV distribution network, the voltage problems did not occur due to the grid being quite strong and therefore resilient. Yearly measurements revealed that the voltage levels throughout the whole year are quite stable. Due to the central location of the storage unit, its impact on voltage levels is only local and cannot impact effectively the voltage levels at the end of the feeders. PV units are distributed among the several feeders and since the storage unit is quite large in capacity, its installation anywhere else than centrally at the transformer station is not really beneficial, since the storage wouldn't be fully utilized and would have an effect only on small part of the network. In discussion with the DSO, who is managing this network, the problem of reverse power flows was highlighted as the focus of the storage operation. Voltage control could be performed at the substation level with the storage unit, where voltage on the secondary side is influenced by the storage operation.

In this paper a Peak Demand Control algorithm is described and further analysed. It comprises 3 key functions stored in the RTU which is controlling the battery. Due to limited technical capabilities of such a unit, optimization process with linear programming and other advanced functionalities cannot be realised on this layer, although there is possibility to perform optimization in the third layer of control. The proposed algorithm is designed to operate locally in the RTU. The PDC algorithm has the following 3 key functions:

- Receiving weather forecast and selection of corresponding transformer power flow profile.

- Calculation of active power flow limit values, where storage starts charging and discharging energy.

- Application of active power threshold in real time.

The first two parts of the control are activated once per day, when forecast update is received, while the third part is performed in real time with predefined time resolution of battery operation. 


\section{A. Weather forecast data analysis}

From the local weather agency, a forecast data of solar irradiation levels is received in $30 \mathrm{~min}$ resolution. Based on the forecast, a cumulative value of daily received solar energy is determined. With the analysis of historical data of solar irradiation levels and transformer power flow measurements for 2015, classified to 16 irradiation levels and for each of them an average transformer active power flow is designated. An average power flow was determined with processing of available measurements of the days in 2015, which received the same amount of solar irradiation as the day for which forecast was received. From predefined database a transformer power flow was designated for the forecasted day.

In Figure 2 we present the borderline transformer active power flow profiles, for the day with highest and lowest irradiation level. Between these two profiles, 14 additional power flow profiles are situated on equally split irradiation level range. The accuracy of the forecast is crucial since it enables dynamic threshold for daily operations. Forecast determines the transformer profile out from the database. Possible inaccurate selection of the profile results in partial coverage of the actual power flows. When these deviate from the forecasted, the storage depletion is faster and consequently peak consumption or production of RES is not fully covered. This can be mitigated with the improved forecasting accuracy, better database of transformer power flow measurements and with possible shortening of the forecasting interval from 24 to 12 hours.

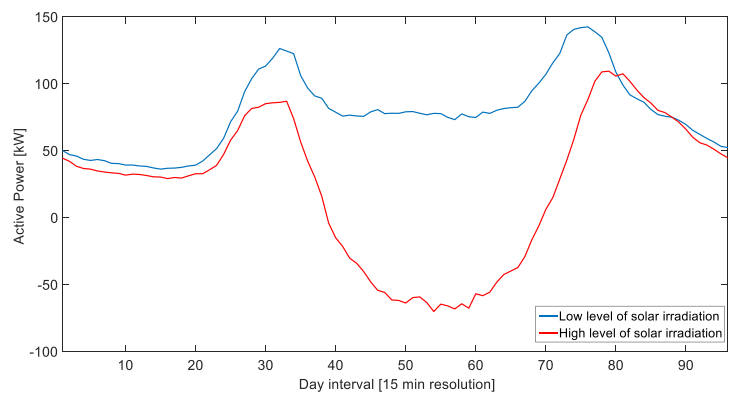

Figure 2: Transformer power flows for day with high level of solar irradiation (red) and day with low level of solar irradiation (blue)

\section{B. Calculation of power flow limit values}

Once the forecasted transformer active power flows are calculated, they are used for calculation of power flow limit levels that define the operation/activation of storage unit. Based on information about initial State of Charge (SoC) level and calculation of SoC throughout the day, an iterative process is performed. In the process we adjust the levels where storage gets activated until we hit SoC limitations or any additional bandwidth conditions are met. In Figure 3 we observe the final scheduled power flow with higher and lower limit value where storage is activated.

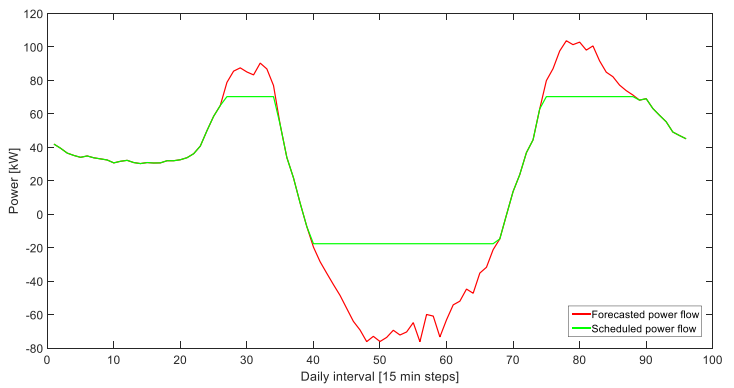

Figure 3: Comparison of forecasted power flow (red) and resulting power flow after batter activation (green)

\section{Real time application of threshold levels}

Once the threshold values are calculated they are used for next 24 hours or until recalculation is performed for operation of the storage in real time. Based on the transformer station measurements, which are available to RTU, storage gets activated and it charges or discharges the amount which surpasses allowed limit of the power flow level. Real-time check of storage availability is performed with monitoring of the actual SoC level of storage. While SoC levels are above minimum and below maximum level, storage will operate as scheduled. Depending on our storage technology we can adjust the resolution of storage operation. Short intervals between storage power input/output adjustments provide smoother peak shaving functionality but impose higher level of stress on the technology. On the other hand, with longer intervals between storage charging/discharging adjustments we decrease stress levels but our control allows higher deviation from threshold levels, which occur within one interval of operation.

\section{SimULATIONS}

For the evaluation of PDC algorithm, a simulation platform was used. It was defined as a combination of Matlab and OpenDSS software. OpenDSS environment was used for definition of the LV grid, transformer substation and running power flow calculation, while Matlab was used for data analysis, definition of control algorithm, storage model and for extraction of power flow results from OpenDSS calculations.

Due to the fact that BESS uses only on transformer power flows for operation and is located next to transformer station, it was possible to simplify the network model to speed up the simulation process as seen in Figure 4. The rest of the LV network model was modelled as a network equivalent load, connected to the transformer bus. To model demand, real measurements of transformer power flows for this network were used. Data was provided by the Slovenian DSO Elektro Gorenjska (EG) which performs monitoring in this network. 


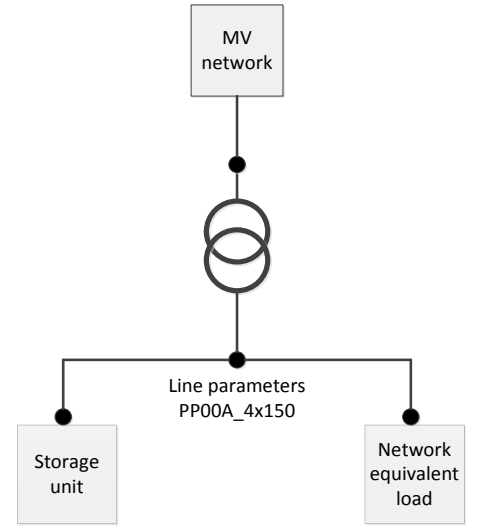

Figure 4: Simplified Network model

Storage model was created in Matlab's Simulink environment, detailed model includes power conversion electronic elements and individual string models for entire storage unit. Rated power of transformer was $400 \mathrm{kVA}$ with rate of $1.001 \%$ load losses and $0.129 \%$ idle losses. Storage unit has $800 \mathrm{~kW}$ rated power of Power Converter Unit (PCU) with available capacity of $660 \mathrm{kWh}$. Losses of the storage model were calibrated using factory test measurements.

\section{RESULTS OF THE SimULATIONS}

Simulations were performed for a weekly interval in summer (week 28), with high levels of solar irradiation and consequentially reverse active power flows in the network. In this section, forecasted data and actual power flows are compared together with SoC levels, both planned and actual, battery schedule and the final active power flows after storage activation.

Figure 5 compares the profile selected based on forecast data and the actual power flow profile in the network for a 4 day interval. Because the predefined profile was determined with average values of multiple days with this level of solar irradiation, the curve is smoother than the actual profile, so this way we only get a general shape of the profile. Partial clouding information is eliminated as we only classify our profiles based on daily values. As a result, we also get deviation in battery SoC which comes out of the scheduling process, compared to actual $\mathrm{SoC}$ in real operation shown in Figure 6.

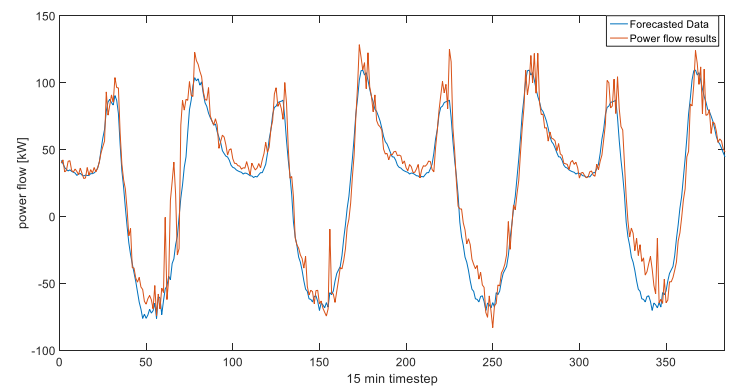

Figure 5: Comparison of forecasted data (blue) and actual power flows (red)

The difference between the calculated and simulated value also occurs due to the fact that in scheduling calculation ideal model was used and battery power input and output were not completely the same in both cases: in simulation, battery charges and discharges with power which was defined with actual power flow and threshold levels as opposed to calculation, where forecast was used. The difference between calculated and simulated value is eliminated before it can affect further calculation. For the calculation of following day, calculation algorithm takes an input actual SoC of the battery, which comes from simulation.

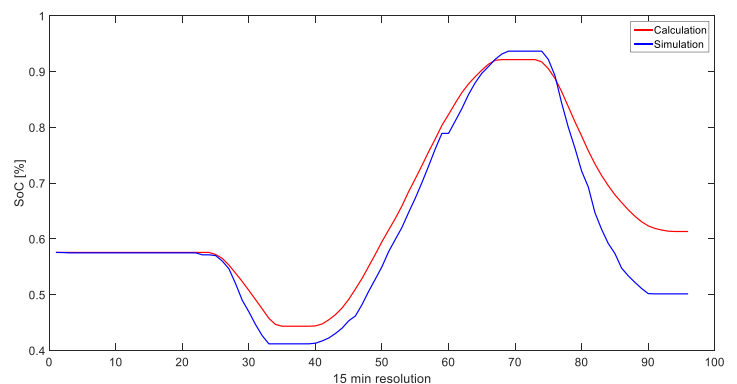

Figure 6: SoC Comparison of calculated (red) and simulated (blue) levels

In Figure 7 comparison of transformer active power flows are compared with and without running a PDC algorithm on implemented storage unit. As it is shown, deviation between forecast and actual power flow can result in only partial shaving of the peak demand interval due to the fact that storage gets depleted sooner than it was supposed to. If we experience cloudy day, which results in sudden drop of solar production with battery operation, we don't mitigate this sudden deviation, due to the fact that it occurs within one interval where storage operates with constant power.

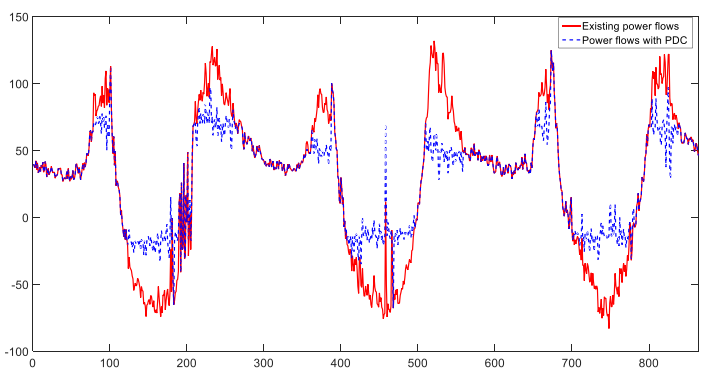

Figure 7: Comparison of existing (red) and storage implemented power flows (blue dashed)

\section{CONCLUSION}

In this paper we presented Peak Demand Control algorithm, used on storage unit in LV distribution network for purposes of peak shaving and peak shifting of transformer active power flows. Based on measurements in transformer station and received solar irradiation forecast, algorithm prepares threshold levels of power flows through transformer, which then activates storage unit to charge or discharge with surplus power in realtime operation. The 'forecasted' transformer power flow profile, which is selected from predefined database, based on solar irradiation level forecast is one of the key components in process. 
If we are able to select a very accurate profile for our calculations, which does not significantly deviate from the real power flows, the algorithm will propose accurate threshold levels. In order to enable good profile selection, a large database of existing profiles is needed, where irradiation levels are divided in very narrow classes, which accurately follow the forecast info. The length of forecast interval has also major role in accuracy. In ongoing work, we plan to implement forecast updating on 12-hour interval or even shorter. With this improvement we will achieve more accurate forecast and based on that more dynamic adjustment of threshold levels for storage operation. Besides the importance of accurate data, simulations also revealed that with more responsive unit, which operates in shorter interval than 15-minute resolution, presented in this paper, we would also be able to achieve smoother resulting power flow.

\section{ACKNOWLEDGMENT}

This work was partially supported by the European Union's Horizon 2020 research and innovation programme, grant agreement No. 646426, project STORY.

\section{REFERENCES}

[1] K. Solangi, M. Islam, R. Saidur, N. Rahim, and H. Fayaz, "A review on global solar energy policy," Renewable and Sustainable Energy Reviews, vol. 15, no. 4, pp. 2149-2163, 2011.

[2] R. Tonkoski, D. Turcotte, and T. H. M. El-Fouly, "Impact of High PV Penetration on Voltage Profiles in Residential Neighborhoods," IEEE Transactions on Sustainable Energy, vol. 3, no. 3, pp. 518-527, 2012.

[3] B. P. Roberts and C. Sandberg, "The Role of Energy Storage in Development of Smart Grids," Proceedings of the IEEE, vol. 99, no. 6, pp. 1139-1144, 2011.

[4] J. M. Eyer, "Electric utility transmission and distribution upgrade deferral benefits from modular electricity storage: a study for the DOE Energy Storage Systems Program.,” Jan. 2009.

[5] J. Tant, F. Geth, D. Six, P. Tant, and J. Driesen, "Multiobjective Battery Storage to Improve PV Integration in Residential Distribution Grids," IEEE Transactions on Sustainable Energy, vol. 4, no. 1, pp. 182-191, 2013.

[6] C. A. Hill, M. C. Such, D. Chen, J. Gonzalez, and W. M. Grady, "Battery Energy Storage for Enabling Integration of Distributed Solar Power Generation," IEEE Transactions on Smart Grid, vol. 3, no. 2, pp. 850-857, 2012.

[7] P. Mercier, R. Cherkaoui, and A. Oudalov, "Optimizing a Battery Energy Storage System for Frequency Control Application in an Isolated Power System," IEEE Transactions on Power Systems, vol. 24, no. 3, pp. 1469-1477, 2009.
[8] A. Zeh and R. Witzmann, "Operational Strategies for Battery Storage Systems in Low-voltage Distribution Grids to Limit the Feed-in Power of Roof-mounted Solar Power Systems," Energy Procedia, vol. 46, pp. 114-123, 2014.

[9] C. J. Bennett, R. A. Stewart, and J. W. Lu, "Development of a three-phase battery energy storage scheduling and operation system for low voltage distribution networks," Applied Energy, vol. 146, pp. 122-134, 2015.

[10] B. Lu and M. Shahidehpour, "Short-Term Scheduling of Battery in a Grid-Connected PV/Battery System," IEEE Transactions on Power Systems, vol. 20, no. 2, pp. 1053-1061, 2005.

[11] O. Megel, J. L. Mathieu, and G. Andersson, "Maximizing the potential of energy storage to provide fast frequency control," IEEE PES ISGT Europe 2013, 2013.

[12] E. Telaretti, G. Graditi, M. Ippolito, and G. Zizzo, "Economic feasibility of stationary electrochemical storages for electric bill management applications: The Italian scenario," Energy Policy, vol. 94, pp. 126-137, 2016

[13] M. J. E. Alam, K. M. Muttaqi, and D. Sutanto, "Mitigation of Rooftop Solar PV Impacts and Evening Peak Support by Managing Available Capacity of Distributed Energy Storage Systems," IEEE Transactions on Power Systems, vol. 28, no. 4, pp. 3874-3884, 2013.

[14] N. Jayasekara, P. Wolfs, and M. A. Masoum, "An optimal management strategy for distributed storages in distribution networks with high penetrations of PV," Electric Power Systems Research, vol. 116, pp. 147-157, 2014.

[15] B. Lu and M. Shahidehpour, "Short-Term Scheduling of Battery in a Grid-Connected PV/Battery System," IEEE Transactions on Power Systems, vol. 20, no. 2, pp. 1053-1061, 2005.

[16] M. Rowe, T. Yunusov, S. Haben, C. Singleton, W. Holderbaum, and B. Potter, "A Peak Reduction Scheduling Algorithm for Storage Devices on the Low Voltage Network," IEEE Transactions on Smart Grid, vol. 5, no. 4, pp. 2115-2124, 2014.

[17] S. Nykamp, A. Molderink, J. L. Hurink, and G. J. M. Smit, "Storage operation for peak shaving of distributed PV and wind generation," 2013 IEEE PES Innovative Smart Grid Technologies Conference (ISGT), 2013.

[18] Y. Yang, H. Li, A. Aichhorn, J. Zheng, and M. Greenleaf, "Sizing Strategy of Distributed Battery Storage System With High Penetration of Photovoltaic for Voltage Regulation and Peak Load Shaving," IEEE Transactions on Smart Grid, vol. 5, no. 2, pp. 982991, 2014.

[19] F. Braam, R. Hollinger, M. L. Engesser, S. Muller, R. Kohrs, and C. Wittwer, "Peak shaving with photovoltaic-battery systems," IEEE PES Innovative Smart Grid Technologies, Europe, 2014.

[20] K. A. Joshi and N. M. Pindoriya, "Day-ahead dispatch of Battery Energy Storage System for peak load shaving and load leveling in low voltage unbalance distribution networks," 2015 IEEE Power \& Energy Society General Meeting, 2015.

[21] S. Karagiannopoulos, A. Rigas, N. Hatziargyriou, G. Hug, and A. Oudalov, "Battery energy storage capacity fading and control strategies for deterministic and stochastic power profiles," 2016 Power Systems Computation Conference (PSCC), 2016. 